\title{
Viabilidade da terapia de aceitação e compromisso para dependentes de drogas
}

\section{Feasibility of acceptance and commitment therapy for drug addicts}

\author{
Pollyanna Santos da Silveira (orcid.org/0000-0002-3010-6477)' \\ Amanda Aparecida Oliveira (orcid.org/0000-0003-1939-0961)2 \\ Jéssica Verônica Tibúrcio Freitas (orcid.org/0000-0001-6071-5970) ${ }^{3}$ \\ Joanna Gonçalves de Andrade Tostes (orcid.org/0000-0001-8392-0352) ${ }^{4}$ \\ Telmo Mota Ronzani (orcid.org/0000-0002-8927-5793) ${ }^{5}$
}

\begin{abstract}
Resumo
A dependência de substâncias é uma das condições de saúde mais estigmatizadas no mundo, contribuindo para o seu agravamento, exclusão social e discriminação. Conscientes da percepção negativa da sociedade em relação à sua condição, essas pessoas podem internalizar o estigma, a partir de crenças, sentimentos e comportamentos negativos sobre si. Uma das intervenções com evidências iniciais positivas para reduzir o estigma internalizado é a Terapia de Aceitação e Compromisso (ACT). Este estudo avaliou a viabilidade de uma intervenção baseada em ACT para reduzir o estigma internalizado entre dependentes de substâncias. Os participantes receberam o tratamento convencional do serviço, além da intervenção composta por seis sessões com periodicidade semanal, conduzidas por uma dupla de terapeutas previamente treinadas. Implementar estratégias para a redução do estigma internalizado pode contribuir para manter os benefícios obtidos ao longo do tempo, aumentando a adesão ao tratamento e a qualidade dos cuidados fornecidos.
\end{abstract}

Palavras-chave: Estigma social. Terapia de aceitação e compromisso. Transtornos relacionados ao uso de substâncias.

\footnotetext{
${ }^{1}$ Universidade Federal de Juiz de Fora (UFJF), Juiz de Fora, Brasil. E-mail: pollyannassilveira@gmail.com.

${ }^{2}$ Universidade Federal de Juiz de Fora (UFJF), Juiz de Fora, Brasil. E-mail: amanda.apa.oliveira@gmail.com.

${ }^{3}$ Universidade Federal de Juiz de Fora (UFJF), Juiz de Fora, Brasil. E-mail:jessicavtiburcio@gmail.com.

4 Universidade Federal de Juiz de Fora (UFJF), Juiz de Fora, Brasil. E-mail: joanna@tostes.org.

${ }^{5}$ Universidade Federal de Juiz de Fora (UFJF), Juiz de Fora, Brasil. E-mail: tm.ronzani@gmail.com.
} 


\begin{abstract}
Substance dependence is one of the most stigmatized health conditions in the world, contributing to its worsening, social exclusion and discrimination. Aware of society's negative perception of their condition, these people can internalize stigma from negative beliefs, feelings and behaviors about themselves. One of the interventions with positive initial evidence to reduce internalized stigma is the Acceptance and Commitment Therapy (ACT). This study evaluated the feasibility of an ACT-based intervention to reduce internalized stigma among substance addicts. The participants received the conventional treatment of the service, in addition to the intervention consisting of six weekly sessions, conducted by a pair of previously trained therapists. Implementing strategies to reduce internalized stigma can contribute to maintaining the benefits obtained over time, increasing adherence to treatment and the quality of care provided.
\end{abstract}

Keywords: Social stigma. Acceptance and commitment therapy. Disorders related to substance use.

O uso de drogas tem sido associado a vários problemas de saúde pública no Brasil e no mundo (Who, 2016; Opas, 2015). Além dos danos físicos e psicológicos relacionados ao consumo abusivo, estudos apontam que a dependência é uma das condições de saúde mais estigmatizadas em todo o mundo, expondo essas pessoas à exclusão social e à discriminação. O estigma envolve consequências diretas no agravamento desse quadro, sendo considerado, por si só, um importante problema de saúde pública (Corrigan et al., 2016; Silveira, Tostes, Wan, Ronzani \& Corrigan, 2018; Schomerus et al., 2011). Tais consequências negativas vão desde a dificuldade em procurar espontaneamente um tratamento até consequências estruturais, que incluem a violação de direitos humanos fundamentais (Rossal, 2018; Who \& Unodc, 2008; Vieira, Caldana \& Corradi-Webster, 2013).

A estigmatização de um comportamento como o uso de drogas pode ser vista como um meio de impor normas sociais para estabelecer limites e comportamentos aceitáveis socialmente (Schomerus et al., 2011). No entanto, estratégias moralizantes associadas a perspectivas proibicionistas têm sido consideradas disfuncionais e pouco efetivas (GomesMedeiros, Faria, Campos \& Tófoli, 2019; Rossal, 2018). Essas estratégias, ao enfatizarem ações relacionadas apenas às características negativas e estigmatizantes do uso de drogas, acabam 
por restringir as possibilidades de acolhimento e acesso para as pessoas com problemas decorrentes desse uso (Room \& Reuter, 2012).

Como consequência, os usuários, ao se tornarem conscientes da percepção negativa e moralizante que a sociedade tem em relação à sua condição, acabam por endossar e internalizar tal visão a partir de crenças, sentimentos e comportamentos negativos sobre si (Corrigan \& Watson, 2002; Boyd, Adler, Otilingam \& Peters, 2013; Silveira et al., 2018). Diversos estudos têm apresentado evidências de que o estigma internalizado está associado a impactos globais, tais como a perda de autoestima e de autoeficácia, diminuindo perspectivas já limitadas de recuperação (Hammarlund, Crapanzano, Luce, Mulligan \& Ward, 2018; Nieweglowski, Dubke, Mulfinger, Sheehan \& Corrigan, 2018; Rusch, Todd, Bodenhausen, Olschewski \& Corrigan, 2010).

Além disso, podem ser identificadas outras consequências negativas entre aqueles que internalizam o estigma, tais como a culpa, a desesperança, a angústia, a autorreprovação e a depressão. Essas consequências, por sua vez, são apontadas como estando associadas a uma rede social mais restrita, à antecipação de rejeição em situações sociais e ao decorrente isolamento social, incluindo o desemprego (Livingston \& Boyd, 2010; Schomerus et al., 2011; Corrigan, Bink, Schmidt, Jones \& Rüsch, 2015).

Apesar da complexidade da internalização do estigma, ainda são escassos os estudos que têm buscado desenvolver e avaliar as estratégias voltadas para a sua redução (Hatzenbuehler, Phelan \& Link, 2013). Ao avaliarem a literatura sobre a efetividade das intervenções para reduzir o estigma associado ao abuso de substâncias, Livingston, Milne, Fang e Amari (2011) apontaram que, entre os estudos cujo foco foi o estigma internalizado, a intervenção baseada na Terapia de Aceitação e Compromisso (ACT) apresentou evidências iniciais de resultados.

A Terapia de Aceitação e Compromisso (ACT) integra a denominada Terceira Onda das Terapias Cognitivo Comportamentais (TCC) (Wilson, Bordieri, Flynn, Lucas \& Slater, 2010). Mais sensível ao contexto e à função dos fenômenos psicológicos, a ACT é uma abordagem clínica 
que pode ser expandida para outros contextos e apresenta como conceito principal a flexibilidade psicológica. Esse conceito é definido como a capacidade de entrar em contato com o momento presente e as experiências internas e, de acordo com o contexto, persistir ou alterar comportamentos na busca por objetivos e valores pessoais. Assim, o foco da ACT é aumentar a flexibilidade psicológica e, para buscá-la, são trabalhados seis processos psicológicos básicos, sendo eles: a aceitação, a desfusão cognitiva, o estar presente, o eu como contexto, os valores e as ações comprometidas (Hayes, Pistorello \& Biglan, 2008; Hayes, 2016).

De acordo com os processos norteadores da ACT, são construídas metas a serem desenvolvidas nas sessões: a) minar o apego do indivíduo à sua agenda comportamental atual; b) mostrar como a agenda não funcional é baseada em controle emocional e em estratégias de evitação; c) ajudar a identificar e a diminuir a fusão cognitiva; d) entrar em contato com um senso de eu que é diferente das reações programadas e das crenças literais sobre si mesmo; e) ajudar o indivíduo a identificar direções de vida por ele valorizadas, juntamente com as metas e as ações necessárias para atingi-las; f) apoiar no engajamento para uma ação comprometida, permitindo que pensamentos, sentimentos e memórias não funcionem como obstáculos, mas como parte esperada de um viver direcionado por metas (Hayes, Strosahl \& Wilson, 1999).

Embora ainda pouco utilizada e difundida para essa finalidade, a ACT para a redução de estigma internalizado foi avaliada no estudo de Luoma, Kohlenberg, Hayes, Bunting e Rye (2008), por meio da aplicação de processos de flexibilidade psicológica, desfusão cognitiva e comportamentos orientados por valores. Os resultados mostraram que a intervenção foi capaz de reduzir a vergonha e o estigma internalizado, além de ter contribuído para uma melhora significativa na autoestima e na saúde mental dos participantes (Luoma et al., 2008). Outro estudo, realizado por Lee, An, Levin e Twohig (2015), apresentou resultados promissores em relação ao uso da $\mathrm{ACT}$, quando comparada às demais intervenções para o tratamento de usuários de substâncias. Os resultados da metanálise mostraram que os efeitos positivos da ACT tenderam a aumentar com o tempo ou se deterioraram mais lentamente que os outros 
tratamentos, ao serem avaliados nas medidas de acompanhamento, follow-up (Lee et al., 2015).

Embora seja notória a complexidade e a extensão dos problemas associados à estigmatização do uso de drogas no cenário brasileiro (Ronzani, Furtado, Higgins-Biddle, 2009; Silveira et al., 2018; Araujo, 2019; Toledo, Góngora \& Bastos, 2017), não há estudos que tenham investigado a viabilidade ou efetividade de estratégias baseadas em ACT para essa finalidade no país. Portanto, o objetivo deste estudo foi avaliar a viabilidade de um protocolo de intervenção baseado nessa abordagem para a redução do estigma internalizado de usuários de substâncias, adaptado pelos autores para o contexto brasileiro.

\section{Método}

Trata-se de um estudo baseado em abordagem qualitativa do tipo descritivo avaliativo. Foram empregados parâmetros para avaliar a aceitação da intervenção, como a frequência de sessões das quais os usuários do serviço participaram e o feedback fornecido por eles e pela equipe de profissionais do serviço. A viabilidade foi avaliada por meio das características do recrutamento, da intervenção e dos resultados alcançados inicialmente (White et al., 2011).

\section{Participantes}

Para compor este estudo, foram convidados a participar usuários de substâncias psicoativas em tratamento no Centro de Atenção Psicossocial ao Usuário de Álcool e outras Drogas (Caps AD III), da cidade de Juiz de Fora, Minas Gerais. Os critérios para participação foram: ter idade igual ou superior a 18 anos, fazer tratamento no Caps AD III e ter disponibilidade de duas horas semanais para participar do grupo.

Os usuários do regime intensivo, que são aqueles cuja presença é diária no serviço, foram convidados pessoalmente a participar do grupo após receberem informações sobre o 
planejamento das atividades. O recurso de inserir os usuários desse regime de tratamento foi uma alternativa viável, tendo em vista que, dessa forma, foram minimizadas perdas de participantes ao longo das sessões. O grupo foi composto de 18 participantes, em média, sendo que esse número flutuou ao longo do processo, considerando que alguns participantes não compareceram a todas as sessões e outros passaram a frequentar o grupo já em andamento.

A idade dos participantes variou de 21 a 56 anos, havendo a predominância de homens, que totalizaram 24 ao longo das sessões, contrapondo-se à presença de um total de apenas oito mulheres. A predominância do gênero masculino nos encontros reflete a realidade do serviço, pois majoritariamente homens acessam o dispositivo. Todas as sessões foram realizadas no interior do Caps AD III em espaço acordado previamente com a coordenação.

\section{Procedimentos}

Ao consentirem em fazer parte da pesquisa, os participantes assinaram um Termo de Consentimento Livre e Esclarecido (TCLE). Todos receberam os recursos terapêuticos convencionais fornecidos pelo serviço, incluindo as consultas com o psiquiatra e a prescrição de medicamentos, quando necessário, o acompanhamento psicológico, individual e em grupo, assim como do serviço social. Adicionalmente, participaram da intervenção para a redução do estigma internalizado, baseada em ACT, composta de seis sessões e com duas horas de duração, com periodicidade semanal, conduzidas e mediadas por uma dupla de terapeutas treinadas previamente. O presente estudo foi aprovado pelo Comitê de Ética em Pesquisa da Universidade Federal de Juiz de Fora (Parecer n. 1.455.439) e todos os parâmetros éticos foram respeitados. 


\section{Protocolo utilizado}

O protocolo do presente estudo foi traduzido e adaptado, pelos próprios autores, a partir do manual Acceptance and Commitment Therapy - Group Therapy Manual for Self-Stigma and Shame in Substance Use Disorder, elaborado por Luoma, Kohlenberg e Hayes (2005). Esse protocolo foi selecionado para este estudo devido ao fato de ter sido aplicado na mesma população-alvo em outro país, apresentando evidências iniciais positivas (Luoma et al., 2008).

O manual inclui uma introdução sobre o estigma internalizado e social, aspectos relacionados à desfusão cognitiva e à flexibilidade comportamental, à aceitação e ao controle de emoções e pensamentos, bem como conteúdos referentes às ações orientadas pelos valores. As sessões incluem discussões e exercícios vivenciais produzidos para ajudar os participantes a enxergarem quão natural é ter pensamentos estigmatizantes e tentar controlá-los, evitá-los ou se livrar deles. Por meio dos exercícios, os participantes foram orientados sobre como deixar para trás ligações ao conteúdo literal de pensamentos e emoções estigmatizantes. As etapas do protocolo foram: a) aumentar a consciência experimental e intelectual do estigma social, do estigma internalizado, da vergonha e de seus efeitos; b) aumentar a consciência experimental do paradoxo da supressão de pensamentos; c) introduzir a desfusão cognitiva; d) introduzir o conceito de que o controle é o problema e que tentar controlar os pensamentos e sentimentos aversivos é parte das estratégias de defesa; e) criar uma flexibilidade comportamental, empática e aceitável por meio da introdução e do compartilhamento de experiências desconfortáveis com o grupo; e f) durante o uso de experiências e metáforas, ajudar os participantes a definir seus valores e objetivos, além de se comprometer para uma mudança comportamental. 


\section{Análise das informações}

As informações qualitativas coletadas a partir das observações das sessões e dos registros de campo foram analisadas a partir dos seguintes parâmetros, indicados por White et al. (2011) acerca da viabilidade e da aceitação de intervenções baseadas em ACT:

a) População: os usuários puderam ser identificados e recrutados apropriadamente para uma intervenção baseada em ACT para a redução de estigma?

b) Intervenção: a ACT seria uma intervenção aceitável para indivíduos com dependência de substâncias? Eles foram capazes de identificar os valores e estabelecer metas para o tratamento? Eles compreenderam o conceito de estigma internalizado e conseguiram identificar estratégias?

c) Resultados: quais medidas seriam importantes para avaliar o efeito da ACT na redução do estigma internalizado de dependentes de substâncias?

\section{Resultados e discussão}

Diferentemente da versão original do protocolo de intervenção, que prevê três sessões, as atividades desenvolvidas com o grupo ocorreram em seis sessões. O aumento no número de sessões justificou-se em função da própria dinâmica do grupo e das adaptações necessárias para que cada atividade cumprisse seu objetivo, sem que houvesse dispersão dos participantes. Os temas de cada uma das sessões estão descritos na Figura 1. 
Figura 1. Descrição dos temas desenvolvidos em cada sessão do protocolo

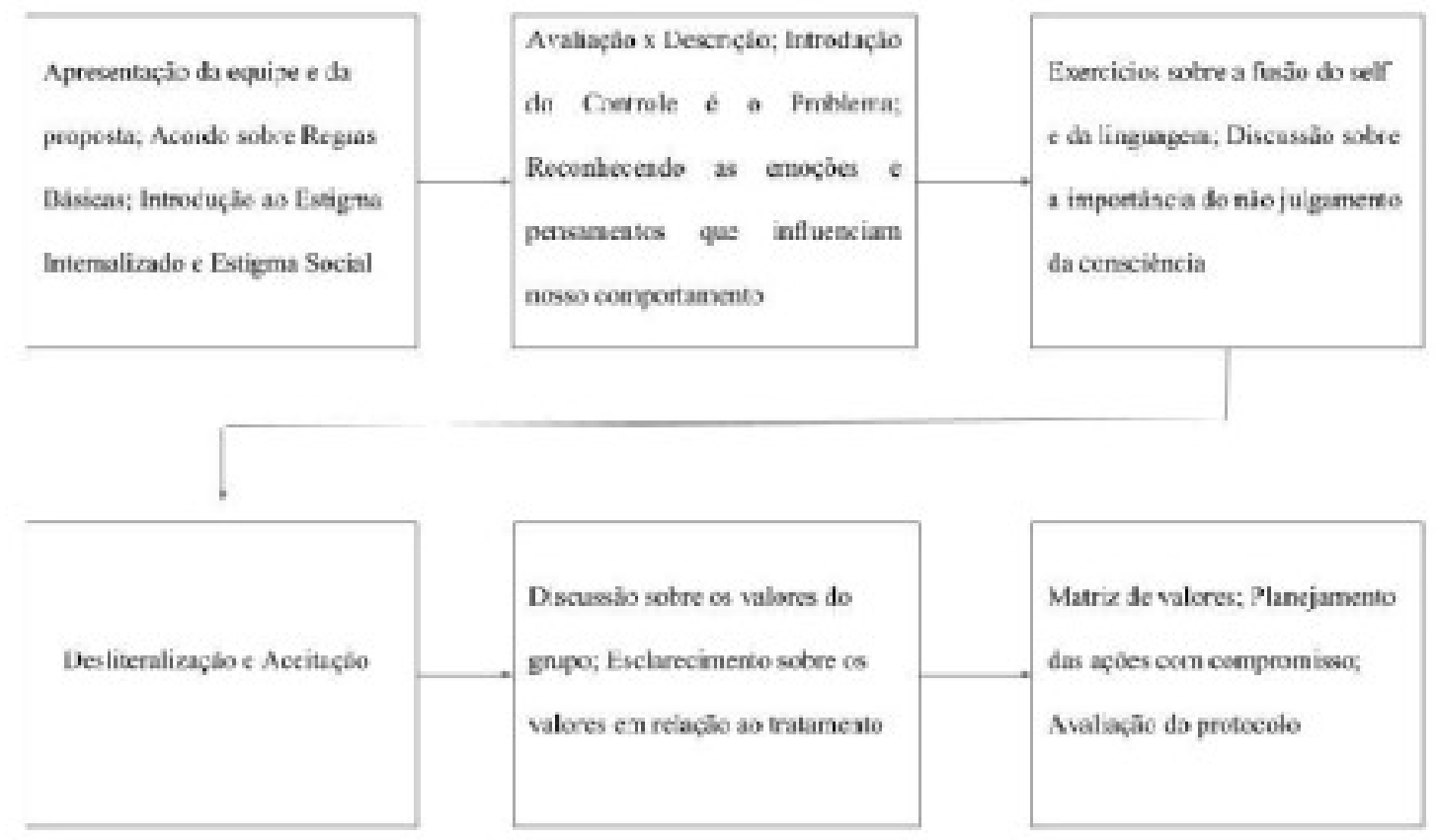

Fonte: Fluxograma elaborado pelos autores.

\section{Sessões}

A primeira sessão teve início com as apresentações individuais dos 23 usuários presentes, da equipe do estudo e das terapeutas mediadoras, do conteúdo e dos objetivos do grupo. Algumas questões levantadas foram: "Por que estou participando do grupo?" e "O que eu gostaria de aprender com o grupo?" Para criar um ambiente acolhedor e de confidencialidade, o próprio grupo estabeleceu regras: falar de forma objetiva, levantar a mão para falar, falar a verdade, ser honesto, manifestar-se no caso de uma situação desconfortável, em vez de apenas sair do grupo.

O tema estigma foi abordado a partir de rótulos de produtos comercializados, tendo em vista que frequentemente são utilizadas as marcas comerciais para fazer referência a diversos produtos. Os usuários apontaram que o uso de drogas muitas vezes é alvo de rotulação. Alguns 
relatos demonstraram tal processo, acarretando na desvalorização tanto pessoal quanto profissional decorrente do uso de drogas. Algumas expressões estigmatizantes foram utilizadas com relação ao consumo de álcool, como: "pinguço", "cachaceiro", "bêbado", "bebum", "sem vergonha", "cobra de vidro". Em relação ao uso de crack, os participantes afirmaram que há uma maior discriminação quando comparada ao uso de álcool. Alguns relatos sinalizam a percepção de discriminação, tais como: "acha que é ladrão", "chamam de verme", "cracudo", "noiado", "chama de tudo, menos de ser humano". Além disso, os usuários apontaram que a própria família culpabiliza os dependentes pelo uso e pela condição em que se encontram, não havendo uma relação de confiança. Os usuários afirmaram, ainda, que "os usuários de álcool discriminam quem fuma maconha, quem fuma maconha discrimina quem usa cocaína e quem usa cocaína discrimina quem usa crack". Para eles, a estigmatização está associada à ocultabilidade da dependência, o que pode ser visto no relato de que "a pessoa que usa cocaína consegue disfarçar, esconder, mas quem usa álcool ou crack fica marcada. No caso do álcool, é difícil esconder, dá pra ver na sua cara que você tá bêbado". Por fim, destacou-se entre as falas o processo de naturalização da discriminação na afirmação: "com o passar do tempo, as ofensas viram rotina e você acaba se acostumando".

Na segunda sessão, o grupo contou com 20 participantes. Retomou-se o objetivo do encontro anterior e um dos usuários comparou o estigma a uma marca semelhante à sinalização feita a ferro nos bois. Na avaliação e classificação das emoções experienciadas, os participantes nomearam como emoções boas a alegria, felicidade, euforia, o medo em alguns momentos e a saudade. Por outro lado, entre as emoções ruins, destacaram-se a tristeza, raiva, ódio, frustração, ciúme, inveja e o arrependimento. No encerramento, aplicou-se a "metáfora passageiros no ônibus", amplamente utilizada na ACT, a fim de trabalhar a aceitação de emoções e pensamentos negativos. A metáfora consiste na ideia de que todo indivíduo é o motorista de seu próprio ônibus, sendo a placa do ônibus os valores e os passageiros são os pensamentos, sentimentos, estados corporais, memória e outros aspectos da experiência. Existem passageiros bons e outros indesejados e, em algumas situações, os indesejados 
buscam pegar o controle do ônibus, mas ressalta-se a possibilidade de retomar o controle da situação e criar estratégias, por exemplo, aceitar a presença desses passageiros, entendendo que mesmo sendo ameaçadores o controle não está com eles.

No terceiro encontro, 16 participantes estavam presentes. Uma meditação foi realizada no início, sendo avaliada pelos usuários de forma positiva, a partir de falas como: "dá uma leveza". A "metáfora do ônibus" foi relembrada a respeito dos pensamentos bons e ruins, sendo enfatizado que, mesmo ruins, são apenas pensamentos. Embora não seja possível controlar os pensamentos, os comportamentos podem ser manejados. Utilizou-se a técnica de desliteralização, que consiste em reduzir um pensamento estigmatizante a apenas uma palavra, ressaltando que palavras são apenas sons. Inicialmente, para demonstração, a técnica foi empregada com a palavra "leite" e, na sequência, seguindo sugestão dos participantes do grupo, utilizou-se a palavra "verme". Os participantes perceberam que uma palavra tão ofensiva de ser ouvida, ao ser repetida diversas vezes em alta voz, perde o significado atribuído de forma pejorativa.

$\mathrm{Na}$ quarta sessão, a dupla de terapeutas retomou os objetivos dos últimos três encontros com os 18 participantes presentes. Foi proposto para exercício que cada um escrevesse em um crachá uma característica própria que mudaria se pudesse. Cada participante descreveu as próprias sensações, emoções e pensamentos vivenciados, além do peso que esses rótulos têm, principalmente, nas situações em que estão expostos: "não andaria com isso na rua", "me sinto muito exposta". Para finalizar, foi utilizada a "metáfora da lápide", iniciando o tema acerca dos valores e solicitando que falassem de que forma gostariam de ser lembrados depois que morressem, o que gostariam que estivesse escrito na lápide, relacionando os valores com as ações em vida. Entre os relatos citados, destacaram-se ser lembrados "como uma pessoa animada", "alguém que sempre ajudou os outros" e uma pessoa que "nunca fez mal a ninguém". Contrapondo-se a esses relatos, é interessante notar que o uso de drogas e o rótulo de usuário foi citado como sendo um dos que eles não gostariam de ser lembrados. 
O quinto encontro, contando com 13 participantes, teve início com um relato mencionando sentimentos negativos ligados à abstinência, no qual um participante denominou a estratégia utilizada para enfrentar os sintomas com um "tipo uma fuga", "fugi de mim mesmo" e, a seguir, mencionou: "eu acho que consegui pegar a direção do meu ônibus". Após o relato, teve início a atividade de meditação como uma forma de retomar a atenção para o momento presente, um dos processos que constituem a ACT. O tema desse encontro abrangeu as dificuldades enfrentadas no tratamento e o que já foi recuperado por meio disso, a partir das seguintes perguntas: "O que eu quero com meu tratamento?" Para onde eu quero que o ônibus vá?" "Quais são as barreiras dentro de mim mesmo para avançar nessa direção?" "O que eu tenho feito com essas barreiras?" "O que isso tem me custado?" Realizou-se uma diferenciação de objetivo e valor, na qual o objetivo tem uma possível conclusão e, após o seu término, é substituído por outros objetivos. De modo distinto, os valores estão relacionados ao ser e àquilo que direciona a vida do indivíduo.

Por fim, no sexto e último encontro, 17 integrantes estavam presentes. O encontro teve início com uma estratégia de meditação e, em seguida, os valores foram relembrados por meio da "matriz de valores", em que o indivíduo pode ser aproximar ou se afastar dos valores estabelecidos de forma individual. Comportamentos de aproximação desses valores consistem em ações com compromisso; em contrapartida, comportamentos de afastamento desses valores podem gerar sentimentos desagradáveis, nessa etapa são evidenciados os comportamentos de manejo. Foi trabalhado, nessa etapa, que o uso de drogas funciona como um comportamento de manejar as frustrações, a partir de relatos como: "bebo, fumo e choro". No final do encontro, um dos usuários propôs que todos os participantes escrevessem em um papel o que desejavam descartar. Entre as anotações, foram frequentes as que mencionaram que eles gostariam de ficar livres de mágoas, da intolerância, do crack, da irresponsabilidade, da ilusão, dos pensamentos ruins, da bebida, da tristeza por ter perdido a mãe, da infelicidade, do alcoolismo, da desconfiança das pessoas, da ignorância, dos vícios, das drogas e do cigarro. 


\section{Avaliação}

Seguindo os parâmetros indicados e supracitados, a viabilidade da intervenção foi avaliada com base na população, na intervenção e nos resultados. Os indicadores relacionados à população se concentraram na identificação e no recrutamento dos usuários do serviço, com o objetivo de participarem da intervenção baseada em ACT para redução de estigma. De modo geral, essa seleção foi acessível, embora possa ser destacada a dificuldade encontrada com a primeira estratégia utilizada. Inicialmente, foram convidadas as pessoas que buscaram o tratamento no serviço durante o mês da seleção, tendo sido feito contato telefônico posterior para agendar o início do grupo. No entanto, dos 52 usuários convidados, apenas um compareceu ao grupo no dia e horário fixado. Uma das explicações possíveis apontadas pela coordenadora do serviço é que, em decorrência dos desafios inerentes ao tratamento de dependentes de substâncias, há um alto índice de abandono no primeiro mês de tratamento. Como alternativa, a coordenadora e os profissionais do serviço sugeriram às terapeutas e aos demais integrantes da equipe que convidassem os usuários em regime intensivo.

A aceitabilidade da intervenção pelos usuários do serviço e pela equipe de profissionais presentes foi alta, não tendo sido mencionados aspectos negativos ao longo dos encontros e no término das atividades. Na avaliação do protocolo, os usuários relataram que as sessões do grupo foram agradáveis, construtivas, interessantes, e que, a partir do grupo, tiveram a oportunidade de "mostrar um outro lado deles". Foi possível observar que alguns dos processos de estigmatização passaram a ser compreendidos pelos participantes por meio de algumas afirmações, tais como: "eu não sou um rótulo de cachaça" e "não está escrito 51 na minha testa". Os participantes mencionaram que aprenderam sobre o estigma, "sobre o que as outras pessoas pensam deles". Além disso, os participantes compreenderam a definição de valor, como "ser responsável”, "assertivo", "ser uma pessoa boa", assim como os sentimentos trazidos quando se distanciam dos valores, "triste", "frustrada", "culpa", "coração vazio". Foram citados

como comportamentos de manejo desses problemas: "me afastar das pessoas", "dar um 
passeio" e "ficar deitada no sofá", contrapondo-se às estratégias comumente utilizadas como "quando estou triste eu bebo, fumo e choro". Entre os comportamentos de aproximação, destacou-se "pedir desculpas".

Os participantes avaliaram o método e as atividades como interessantes e diferentes das que já haviam participado, destacando as metáforas como um recurso de fácil compreensão, ressaltando a "metáfora do ônibus", anteriormente descrita. Com relação à duração de cada encontro e do protocolo, verificou-se que os objetivos propostos e a sua execução foram adequados. Entre os relatos, destacaram-se aqueles de que "o tempo passou rápido", descrevendo não somente o quanto foi agradável, mas ressaltando que o número de encontros poderia ser maior. Um dos participantes nomeou o método utilizado pelas terapeutas como um "método de escuta ativa", avaliando de forma positiva.

As terapeutas questionaram os participantes sobre possíveis benefícios por meio dos encontros no grupo, observando, assim, indicadores de redução do estigma internalizado. Alguns dos relatos foram: "depois do grupo não estou dando tanta atenção para o que os outros estão falando", "me sinto mais relaxado, tranquilo e pensando mais". Uma participante mencionou que o grupo a ajudou a enfrentar situações difíceis, como lidar com a morte de sua mãe. A partir da proposta da ACT, os participantes relataram que se sentiram encorajados a não evitar ou controlar pensamentos e sentimentos, sobretudo, nos seguintes relatos: "eu posso", "peguei o volante do ônibus de novo" e "eu vivi a emoção, eu não fugi, ainda que seja doloroso".

Podemos destacar que mesmo com os desafios no recrutamento, a adesão dos participantes foi alta. Com exceções de participantes que estavam no serviço para atendimento e aproveitavam a oportunidade de participar do grupo de maneira isolada, um grupo com 13 indivíduos tiveram presença assídua nos encontros. Esses participantes também contribuíram intensamente no desenvolvimento da proposta e continuidade das sessões. 


\section{Considerações finais}

A intervenção desenvolvida neste estudo teve como foco as mudanças experimentais e contextuais que, aliadas ao uso de estratégias didáticas, possibilitaram aos participantes o desenvolvimento de um repertório mais amplo, flexível e efetivo para lidar com problemas específicos (Hayes, Luoma, Bond, Masuda \& Lillis, 2006). Os efeitos das sessões sobre os participantes mostraram que o uso do protocolo baseado em ACT para a redução do estigma internalizado entre dependentes de substâncias mostrou-se viável. Além disso, o feedback positivo dos participantes e os relatos durante as sessões evidenciam a aplicabilidade desse tipo de intervenção no serviço de saúde mental, conforme recomendado por Corrigan (2004).

Segundo Donabedian (2003), a aceitabilidade de uma intervenção em saúde pelo público-alvo a quem foi destinada, assim como pelos demais profissionais envolvidos no tratamento, é um dos importantes atributos indicativos de sua qualidade. Em consonância com esses achados, na avaliação da dinâmica do processo, destacaram-se o estabelecimento de um vínculo forte no grupo, o respeito dos usuários em ouvir uns aos outros e participar das atividades propostas pelas terapeutas mediadoras. Houve uma grande aceitação dos usuários e dos profissionais que acompanharam todo o processo. Apesar das dificuldades iniciais do recrutamento, o protocolo mostrou-se satisfatoriamente ajustável à rotina do serviço de saúde, podendo ser enfatizada a adesão dos usuários às atividades desenvolvidas, em termos do número de participantes em cada sessão e da qualidade de seu envolvimento.

Devido ao fato de ser um estudo de viabilidade, podem ser apontadas algumas limitações, assim como perspectivas futuras de aplicação da intervenção em outros grupos terapêuticos. No que tange aos participantes, como o grupo era aberto, houve variação durante as sessões, ausência de alguns membros que participavam ativamente e a inserção de novos. Devido à própria condição de saúde e à dificuldade de adesão ao tratamento, alguns usuários do serviço faltaram no dia em que ocorreram as sessões e, com isso, perderam temas e atividades trabalhados que poderiam ser benéficos para eles. Espera-se que, a partir da 
intervenção realizada no presente estudo e de seus resultados favoráveis, possam ser implementados outros grupos terapêuticos baseados em ACT nos serviços de saúde mental. Recomenda-se que novas avaliações sejam feitas por meio de métodos randomizados para acrescentar maior rigor no controle de variáveis interferentes.

\section{Fonte de financiamento}

O estudo foi financiado pela Fundação de Amparo à Pesquisa do Estado de Minas Gerais (FAPEMIG), por meio dos processos BDP-00678-14 e PPSUS AQP-03256-13; Conselho Nacional de Desenvolvimento Científico e Tecnológico (CNPq), por meio do Edital Universal 439408/2018-0. O co-autor Telmo Mota Ronzani recebeu bolsa de produtividade 1D do CNPq.

\section{Referências}

Araujo, F. S. (2019). Quebrando estigmas: uma alternativa ao proibicionismo das drogas por meio da redução de danos. Resenha: Jamais fomos zumbis: contexto social e craqueiros na cidade de São Paulo. Alves YDD. Salvador: EDUFBA; 2017. Cadernos de Saúde Pública, 35(3), e00014019. doi: 10.1590/0102-311X00014019.

Boyd, J. E., Adler, E. P., Otilingam, P. G, \& Peters, T. (2013). Internalized Stigma of Mental Illness (ISMI) Scale: A Multinational Review. Comprehensive Psychiatry, 55, 221-231. doi: 10.1016/j.comppsych.2013.06.005.

Corrigan, P. W. (2004). How Stigma Interferes with Mental Health Care. American Psychologist, 59(7), 614-625.

Corrigan, P. W., Bink, A. B., Schmidt, A., Jones, N., \& Rüsch, N. (2015). What is the Impact of Self-Stigma?: Loss of Self-Respect and the "Why Try" Effect. Journal of Mental Health, 25(1), 10-15. doi: 10.3109/09638237.2015.1021902. 
Corrigan, P. W., Schomerus, G., Shuman, V., Kraus, D., Perlick, D., Harnish, A., \& Smelson, D. (2016). Developing a Research Agenda for Understanding the Stigma of Addictions. Part I: Lessons from the Mental Health Stigma Literature. American Journal on Addictions, 26, 59-66. doi: 10.1111 /ajad.12458.

Corrigan, P. W., \& Watson, A. C. (2002). The Paradox of Self-Stigma and Mental Illness. Clinical Psychology Science and Practice, 9(1), 35-53.

Donabedian, A. (2003). An Introduction to Quality Assurance in Health Care. Oxford: Oxford University Press.

Gomes-Medeiros, D., Faria, P. H., Campos, G. W. S., \& Tófoli, L. F. (2019). Drug Policy and Collective Health: Necessary Dialogues. Cadernos de Saúde Pública, 35(7), e0024261 8. doi: $10.1590 / 0102-311 \times 00242618$.

Hammarlund, R. A., Crapanzano, K. A., Luce, L., Mulligan, L. A., \& Ward, K. M. (2018). Review of the Effects of Self-Stigma and Perceived Social Stigma on the Treatment-Seeking Decisions of Individuals with Drug - and Alcohol - Use Disorders. Substance Abuse and Rehabilitation, 9, 115-136. doi:10.2147/sar.s183256.

Hatzenbuehler, M. L., Phelan, J. C., \& Link, B. G. (2013). Stigma as a Fundamental Cause of Population Health Inequalities. American journal of public health, 103(5), 813-821.

Hayes, S. C. (2016). Acceptance and Commitment Therapy, Relational Frame Theory, and the Third Wave of Behavioral and Cognitive Therapies - Republished Article. Behavior Therapy, 47(6), 869-885. doi:10.1016/j.beth.2016.11.006.

Hayes, S. C., Luoma, J. B., Bond, F. W., Masuda, A., \& Lillis, J. (2006). Acceptance and Commitment Therapy: Model, Processes and Outcomes. Psychology Faculty Publications, Paper 101, 130. Retrieved from http://scholarworks.gsu.edu/psych_facpub/101. 
Hayes, S. C., Pistorello, J., \& Biglan, A. (2008). Terapia de aceitação e compromisso: modelo, dados e extensão para a prevenção do suicídio. Revista Brasileira de Terapia Comportamental e Cognitiva, 10(1), 81-104. doi:10.31505/rbtcc.v10i1.234.

Hayes, S. C., Strosahl, K. D., \& Wilson, K. G. (1999). Acceptance and Commitment Therapy. An Experimental Approach to Behavior Change. New York: Guilford Press.

Lee, E. B., An, W., Levin, M. E., \& Twohig, M. P. (2015). An Initial Meta-Analysis of Acceptance and Commitment Therapy for Treating Substance Use Disorders. Drug and Alcohol Dependence, 155, 17. doi: 10.1016/j.drugalcdep.2015.08.004.

Livingston, J. D., \& Boyd, J. E. (2010). Correlates and Consequences of Internalized Stigma for People Living with Mental Illness: A systematic Review and Meta Analysis. Social Science and Medicine, $71(12), 2150-2161$.

Livingston, J. D., Milne, T., Fang, M. L., \& Amari, E. (2011). The Effectiveness of Interventions for Reducing Stigma Related to Substance Use Disorders: A Systematic Review. Addiction, $107(1), 39-50$.

Luoma, J. B., Kohlenberg, B. S., \& Hayes, S. C. (2005). Acceptance and Commitment Therapy Group: Therapy Manual for Self-Stigma and Shame in Substance Use Disorder.

Luoma, J. B., Kohlenberg B. S., Hayes S. C., Bunting K., \& Rye, A. K. (2008). Reducing Self Stigma in Substance Abuse through Acceptance and Commitment Therapy: Model, Manual Development, and Pilot Outcomes. Addiction Research and Theory, 16(2), 149-165.

Nieweglowski, K., Dubke, R., Mulfinger, N., Sheehan, L., \& Corrigan, P. W. (2018). Understanding the Factor Structure of the Public Stigma of Substance Use Disorder, Addiction Research \& Theory, 1-7. doi: 10.1080/16066359.2018.1474205. 
Organização Pan Americana de Saúde [OPAS]. (2015). Informe sobre la situación regional sobre el alcohol y las aludenlas Américas. Washington, DC: OPAS.

Ronzani, T. M., Furtado, E. F., \& Higgins-Biddle, J. (2009). Stigmatization of Alcohol and Other Drug Users by Primary Care Providers in Southeast Brazil. Social Science \& Medicine, 69(7), 1080-1084.

Room, P., \& Reuter, P. (2012). How Well do International Drug Conventions Protect Public Health?. Lancet, 379, 84-91.

Rossal, M. (2018). Social Effects of Prohibitionism in the Americas and New Drug Policies. (3, 27-45). In T. M. Ronzani (Org.). Drugs and Social Context: Social Perspectives on the Use of Alcohol and Other Drugs. Cham: Springer.

Rüsch, N., Todd, A. R., Bodenhausen, G. V., Olschewski, M., \& Corrigan, P. W. (2010). Automatically Activated Shame Reactions and Perceived Legitimacy of Discrimination: A Longitudinal Study among People with Mental Illness. Journal of Behavior Therapy and Experimental Psychiatry, 41(1), 60-63. doi:10.1016/j.jbtep.2009.10.002.

Schomerus, G., Corrigan, P. W., Klauer, T., Kuwert, P., Freyberger, H. J., \& Lucht, M. (2011). SelfStigma in Alcohol Dependence: Consequences for Drinking Refusal Self-Efficacy. Drug and Alcohol Dependence, 114(1), 12-17. doi: http://dx.doi.org/10.1016/j.drugalcdep.2010.08.013.

Silveira, P. S., Casela, A. L. M., Monteiro, É. P., Ferreira, G. C. L., Freitas, J. V. T., Machado, N. M., Noto, A. R., \& Ronzani, T. M. (2018). Psychosocial Understanding of Self-Stigma among People who Seek Treatment for Drug Addiction. Stigma and Health, 3(1), 42-52. doi:10.1037/sah0000069.

Silveira, P. S., Tostes, J. G. A., Wan, C., Ronzani, T. M., \& Corrigan, P. W. (2018). The Stigmatization of Drug Use as Mechanism of Legitimation of Exclusion (1, 15-25). In T. 
M Ronzani (Org.). Drugs and Social Context: Social Perspectives on the Use of Alcohol and Other Drugs. Cham: Springer.

Toledo, L., Góngora, A., \& Bastos, F. I. P. M. (2017). À margem: uso de crack, desvio, criminalização e exclusão social - uma revisão narrativa. Ciência \& Saúde Coletiva, 22(1), $31-42$.

Vieira, F. S., Caldana, R. H. L., \& Corradi-Webster, C. M. (2013). Relatos de auxiliares de enfermagem psiquiátricos sobre suas práticas e sobre pessoas internadas por uso compulsivo de substâncias psicoativas. Saúde e Transformação Social, 4(2), 139-148.

Wilson, K. G., Bordieri M. J., Flynn, M. K., Lucas N. N., \& Slater R. M. (2010). Entendendo a Terapia de Aceitação e Compromisso em Contexto: a história das similaridades e diferenças com outras Terapias Cognitivo Comportamentais. In J. Herbert \& E. Forman (Eds.). Acceptance and Mindfulness in Cognitive Behavior Therapy. Hoboken, NJ: Wiley.

White, R., Gumley, A., McTaggart, J., Rattrie, L., McConville, D., Cleare, S., \& Mitchell, G. (2011). A Feasibility Study of Acceptance and Commitment Therapy for Emotional Dysfunction Following Psychosis. Behaviour Research and Therapy, 49(12), 901-907. doi:10.1016/j.brat.2011.09.003.

World Health Organization [WHO]. (2016). Public Health Dimension of the World Drug Problem. Report by the Secretariat - Executive Board, 140th session, Provisional Agenda Item 10.3, EB140/29. World Health Organization, Geneva. Retrieved from http:// apps.who.int/gb/ebwha/pdf_files/EB140/B140_29-en.pdf.

World Health Organization [WHO] \& United Nations Office on Drugs and Crime [UNODC]. (2008). Principles of Drug Dependence Treatment - Discussion Paper. New York. 